\title{
Rôle de la cueillette, la pêche et la chasse dans l'alimentation au Laos
}

Part of gathering, fishing and hunting in the diet in Laos

\section{Florence Strigler}

\section{(2) OpenEdition}

\section{Journals}

Édition électronique

URL : http://journals.openedition.org/ethnoecologie/907

DOI : 10.4000/ethnoecologie.907

ISSN : 2267-2419

Éditeur

Laboratoire Eco-anthropologie et Ethnobiologie

Référence électronique

Florence Strigler, «Rôle de la cueillette, la pêche et la chasse dans l'alimentation au Laos », Revue d'ethnoécologie [En ligne], 2 | 2012, mis en ligne le 21 décembre 2012, consulté le 01 mai 2019. URL: http://journals.openedition.org/ethnoecologie/907 ; DOI : 10.4000/ethnoecologie.907

Ce document a été généré automatiquement le 1 mai 2019.

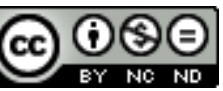

Revue d'ethnoécologie est mis à disposition selon les termes de la licence Creative Commons Attribution - Pas d'Utilisation Commerciale - Pas de Modification 4.0 International. 


\title{
Rôle de la cueillette, la pêche et la chasse dans l'alimentation au Laos
}

\author{
Part of gathering, fishing and hunting in the diet in Laos
}

Florence Strigler

1 Le Laos est l'un des pays les mieux dotés d'Asie du Sud-est en termes de biodiversité ${ }^{1}$ (Duckworth et al 1999). La pauvreté de ses infrastructures et son relatif isolement de l'économie mondiale depuis la fin du XIX ${ }^{e}$ siècle jusqu'à une époque récente, ont protégé ses forêts du pillage des ressources naturelles dont ont été victimes bien d'autres pays. $80 \%$ de la population est rurale et pratique la riziculture. En général, les paysans cultivent aussi quelques légumes, dans des jardins familiaux situés souvent au bord des rivières, ou en association avec le riz dans les essarts ${ }^{2}$. Ils élèvent quelques animaux, principalement volailles et porcins. Ils pratiquent la cueillette tout au long de l'année, mais plus encore en période de soudure ou lorsque les aléas climatiques ont entraîné une mauvaise récolte. Ils pratiquent également la chasse et/ou la pêche. En fonction des villages et des périodes de l'année, les produits sauvages peuvent représenter jusqu'à $80 \%$ des aliments consommés, mis à part le riz (Schlemmer 1999, Clendon 2001).

2 L'agriculture et la chasse-pêche-cueillette sont intimement liées dans la fourniture d'aliments mais aussi dans le mode de vie d'une manière générale. Dans les vallées, les rizières représentent, outre la production de riz, un réservoir d'espèces aquatiques comestibles. Dans les montagnes, l'essartage effectué en rotation permet aux parcelles en friche d'offrir aux villageois des ressources naturelles complémentaires à tous les stades de régénération. Ces pratiques transforment la forêt originelle pour produire des forêts appropriées aux besoins des populations locales (Michon 2004). Réciproquement, l'environnement participe à façonner la culture, notamment alimentaire. Par exemple, l'essartage favorise le développement de forêts de bambou, ce qui a conduit à l'abondance des pousses de bambou dans l'alimentation (Foppes \& Ketphan 2000). 


\section{Le rôle des produits de la cueillette, de la pêche et de la chasse, dans la couverture des besoins nutritionnels}

Dans le passé et dans les différentes régions du monde, les régimes alimentaires traditionnels permettaient en général de couvrir les besoins nutritionnels des populations (Grivetti \& Ogle 2000, Johns \& Sthapit 2004). Les cas de mauvais état nutritionnel étaient dus à des maladies notamment parasitaires, plus qu'à des déficiences d'apports alimentaires (Dounias \& Froment 2006). De nombreux travaux tendent à montrer que la diminution des produits sauvages dans le régime alimentaire s'accompagne en général d'une dégradation de l'état nutritionnel des populations (Pagezy 1993, Kuhnlein \& Receveur 1996, Johns 2003, Frison et al 2005).

4 Ils jouent un rôle important en termes d'apports nutritionnels (Hladik et al 1993, Ogle et al 2001, Burlingame 2000, Kuhnlein 2003) mais aussi d'utilisation des nutriments par l'organisme, par exemple en stimulant l'appétit ou en combattant les infections intestinales (Etkin \& Ross 1994). Étant donné les difficultés de conservation en climat tropical, la majorité des produits (surtout végétaux) sont consommés le jour même. En conséquence, leur valeur nutritionnelle est utilisée de façon optimale, sans pertes liées au stockage et à la conservation.

5 Au Laos, la grande variété des aliments consommés dans les systèmes alimentaires traditionnels contribue (ou contribuait) à apporter les nutriments nécessaires à la santé. Par exemple, chez les Katu, les os et cartilages des animaux sauvages capturés à la chasse étaient écrasés et mis à fermenter dans des tubes de bambou, ce qui constituait une source intéressante de calcium (Krahn 2005). Dans une étude réalisée dans la province de Saravane, Clendon (2001) estime que les produits forestiers apportent $40 \%$ du calcium, $25 \%$ du fer et $40 \%$ des vitamines $A$ et $C$ dans le régime. Nurhasan (2008), dans son étude sur la composition nutritionnelle des espèces animales aquatiques récoltées dans les rizières, montre qu'elles fournissent une grande partie des apports en protéines, calcium, fer et zinc.

6 Notons qu'au Laos, et notamment chez les Lao, les tabous alimentaires sont rares et ne concernent souvent que des périodes précises de la vie des individus (femmes en période post-partum, malades). Sans vouloir adopter une interprétation déterministe de cette observation, on peut dire que la quasi-absence de tabous alimentaires permet d'utiliser pleinement la diversité des aliments disponibles. Les produits animaux et végétaux sont très nombreux, mais souvent en petite quantité et difficiles à se procurer ${ }^{3}$. L'exploitation de l'ensemble des ressources permet en partie de pallier ce problème et d'assurer la sécurité alimentaire.

7 Il est très difficile d'avoir une approche quantitative de la place des produits sauvages dans les apports nutritionnels, étant donné que leur composition nutritionnelle est la plupart du temps inconnue, et que les enquêtes nutritionnelles "oublient" souvent ces produits. Plusieurs causes peuvent expliquer ces "oublis" : certains questionnaires utilisés omettent de poser la question; certains enquêteurs n'interrogent que les ménages habitant à proximité des routes, étant donné la difficulté d'atteindre les villages les plus reculés, qui sont ceux faisant le plus appel aux produits sauvages pour leur subsistance ; les enquêtes sont souvent réalisées à un seul moment de l'année et ne prennent donc pas 
en compte les variations saisonnières, qui sont considérables. Une autre difficulté, à laquelle peut être confronté l'enquêteur ne parlant pas les langues locales, est d'origine linguistique. En lao, la question "qu'avez-vous mangé ?" se dit kin khao kap niang? , ce qui littéralement signifie "vous avez mangé du riz avec quoi ?". En effet, un repas comporte obligatoirement du riz et "manger" au sens de "prendre un repas" se dit kin khao, littéralement "manger du riz". Or, si les produits de la pêche et de la chasse servent effectivement à accompagner le riz, les produits de cueillette sont souvent consommés en dehors des repas. "Manger" ces produits ne se dit alors pas kin khao mais kin lin, littéralement "manger pour jouer". Ces subtilités de vocabulaire peuvent conduire à des erreurs d'estimation dans les enquêtes alimentaires. Au Laos, hormis des enquêtes locales, on ne dispose à l'échelle nationale que des données de la FAO, qui sont calculées à partir des chiffres de la population et ceux de la production minorés des exportations, des déchets, des grains utilisés comme semences, des produits utilisés pour l'alimentation animale, et majorés des importations et de l'aide alimentaire. Les produits sauvages ne sont pas pris en compte, à l'exception des nouvelles enquêtes en cours.

8 Les espèces végétales consommées sont très nombreuses, ainsi que les organes des plantes utilisés : racines, fruits, pousses (de bambou, de rotin), fougères, champignons, feuilles, lianes, moelle (de palmiers, de bananiers), bulbes, fleurs, bourgeons, graines.

Les végétaux féculents comme le taro et l'igname, qui poussent à la période de pré-récolte du riz, viennent souvent se substituer à la céréale durant la période de soudure.

Les pousses de bambou sont consommées en très grande quantité et constituent parfois le seul accompagnement du riz pendant la saison des pluies, voire remplacent le riz pendant la période de soudure.

Photo 1. Pousses de bambou en vente au bord de la route

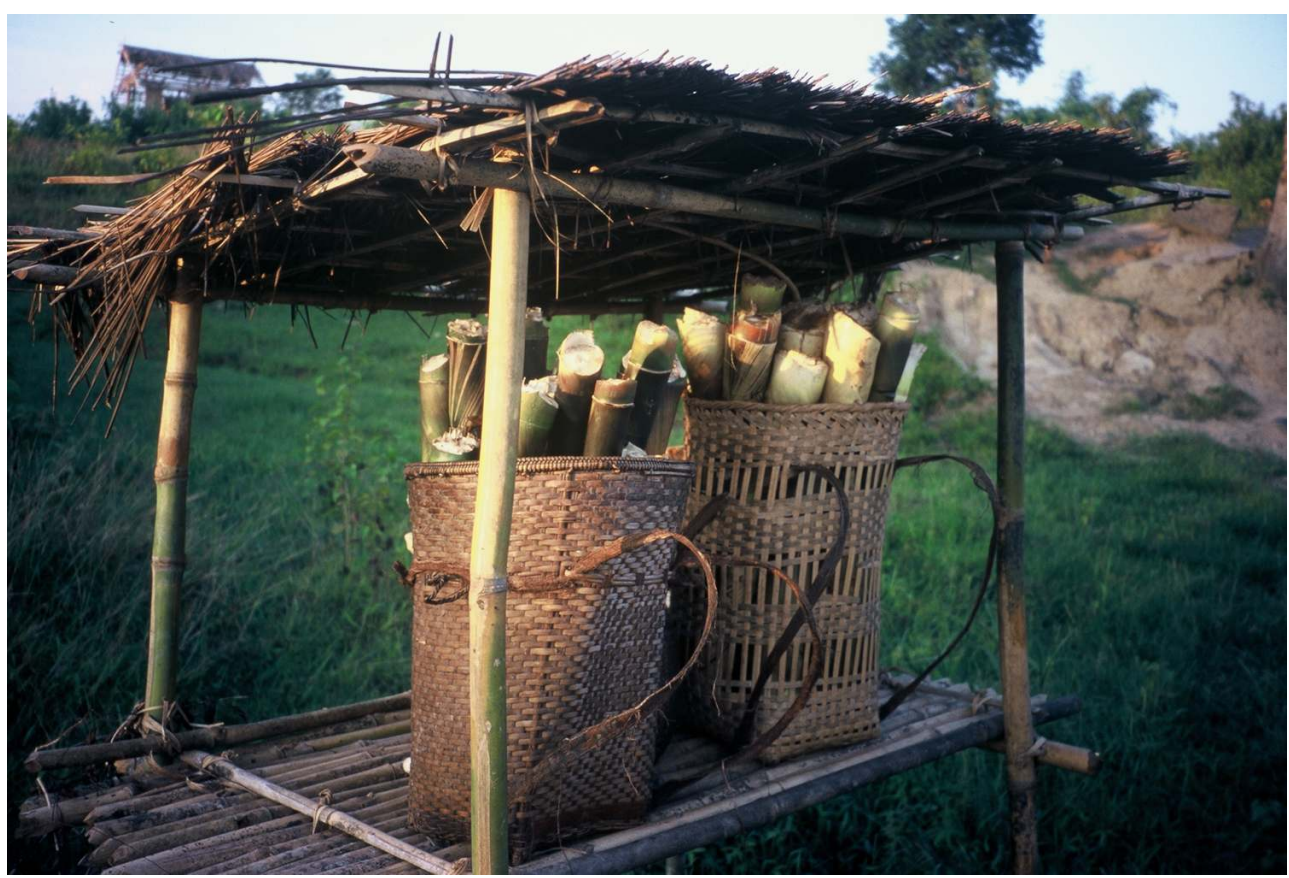

(c) F. Strigler

11 En ce qui concerne les animaux, la chasse au gros gibier a diminué au profit de celle des oiseaux, des petits mammifères (écureuils, porcs-épics, belettes, chauve-souris...) et 
parfois des reptiles. Les insectes font aussi partie fréquemment des repas. DeFoliart (1997) en a dénombré 80 espèces en Thaillande; étant donné la proximité géographique et culturelle entre le Laos et la Thailande, il est probable que ses observations soient transposables au Laos.

12 Près de 250 espèces indigènes de poissons ont été dénombrées au Laos par Davidson en 1975, dont certaines ont disparu. D'autres espèces ont été identifiées depuis : 210 espèces étaient recensées en 1995, 480 en 1999 (Kottelat 2001). Krahn (2005) attire l'attention sur le grand nombre de très petites espèces (inférieures à 6 centimètres), souvent négligées dans les recensements.

13 Pour les populations locales, poissons, batraciens, crustacés, mollusques et insectes aquatiques appartiennent à une même catégorie, celle des "animaux aquatiques" (Nurhasan 2008). Ils jouent un rôle très important dans le régime alimentaire des populations vivant dans les vallées, pour qui ils représentent le principal apport de protéines.

\section{Photo 2. Pêche au carrelet}

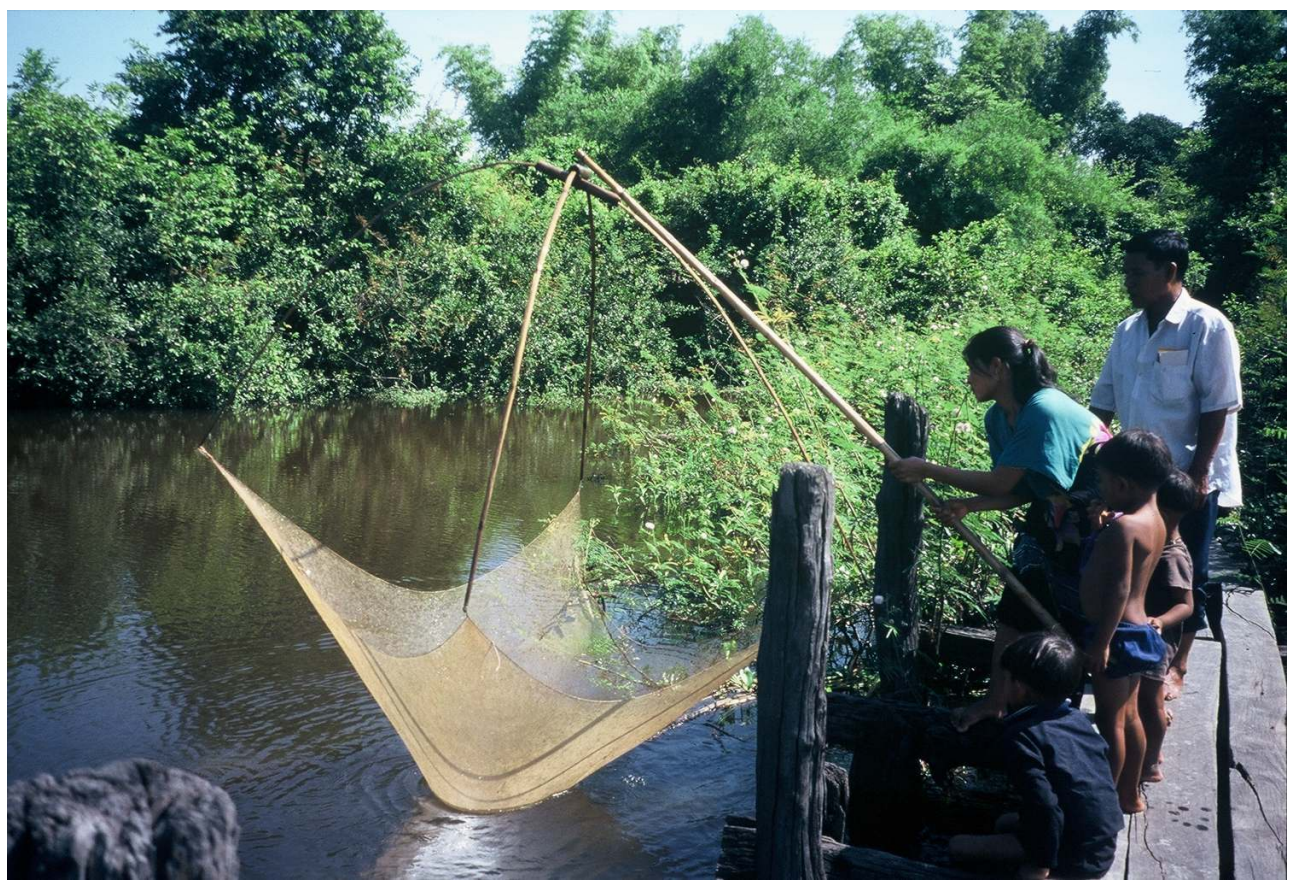

(c) F. Strigler

\section{La forêt et les milieux humides : deux écosystèmes essentiels}

La forêt couvre environ $54 \%$ de la surface du Laos ${ }^{4}$, ce qui est comparable au Cambodge (53\%) mais beaucoup plus que le Vietnam (30\%) ou la Thaïlande (29\%) (FAO 2005). Les hommes y chassent du gros gibier (sangliers, cerfs, chevreuils, etc.), surtout en saison sèche. Les femmes et les enfants chassent des animaux plus petits (écureuils, oiseaux, insectes, etc.) et collectent de nombreux produits végétaux. Les ressources forestières sont cruciales pour la subsistance des populations rurales, mais sont sous-estimées dans les statistiques économiques et les décisions politiques (Emerton 2003). En effet, les 
statistiques officielles prennent en compte uniquement le secteur formel de l'exploitation du bois, mais pas la valeur des produits consommés pour l'autosubsistance, valeur qui échappe au marché et qui est donc invisible pour les décideurs économiques.

Quand on parle de produits sauvages au Laos, on pense avant tout à la forêt. D'ailleurs, "animaux sauvages" se dit en lao sat pa, littéralement "animaux de la forêt", et les légumes sauvages phak pa, "légumes de la forêt". Cela s'explique sans doute par le fait que la forêt occupait autrefois une proportion plus importante du territoire et qu'un village n'était jamais très éloigné d'une forêt. Une autre explication est liée au fait que la forêt est le domaine des esprits, qui incarnent la nature par opposition à la culture, représentée par l'espace villageois (Taillard 1977, Boulbet 1984). C'est ainsi que les grenouilles, par exemple, ne sont pas considérées comme "sauvages", car elles se trouvent dans les rizières, qui sont des espaces humanisés.

Pour les Laotiens, les produits de la forêt sont dotés de propriétés exceptionnelles, notamment d'une valeur nutritionnelle supérieure aux plantes et animaux domestiqués, et de vertus médicinales. Ils comportent une énergie vitale, du fait qu'ils proviennent de la nature, source de vie. La forêt, ou autrement dit la nature, représente la longévité, la prospérité, le bonheur et la force, autant de biens que les hommes sollicitent des esprits, en échange de biens de culture comme le riz, l'alcool, le tabac, les attitudes de déférence ${ }^{5}$ (Doré 1996).

17 Ce sont plutôt les populations austro-asiatiques et miao-yao qui vivent dans les régions les plus boisées. Cependant, les produits forestiers sont extrêmement appréciés également par les Lao, qui les leur achètent. En témoignent le succès du marché dong mak khay, à 16 kilomètres de la capitale Vientiane, et du marché hmong du kilomètre 52, spécialisés dans les produits "sauvages".

18 Les milieux humides (rizières, rivières, étangs, marécages) représentent d'autres écosystèmes ${ }^{6}$ très riches en produits végétaux et animaux utilisés pour l'alimentation. Les rizières sont un réservoir d'espèces aquatiques nombreuses (Halwart 2006) : poissons, grenouilles, crabes, crevettes, escargots, mais aussi de rongeurs : rats, taupes, hérissons, etc. Les rivières recèlent quantité de poissons et autres espèces aquatiques, ainsi que d'algues comestibles. Quant aux étangs et marécages, ils fournissent aussi des plantes comestibles comme le lotus ou le liseron d'eau. 
Photo 3. Les rizières inondées, un réservoir d'espèces comestibles

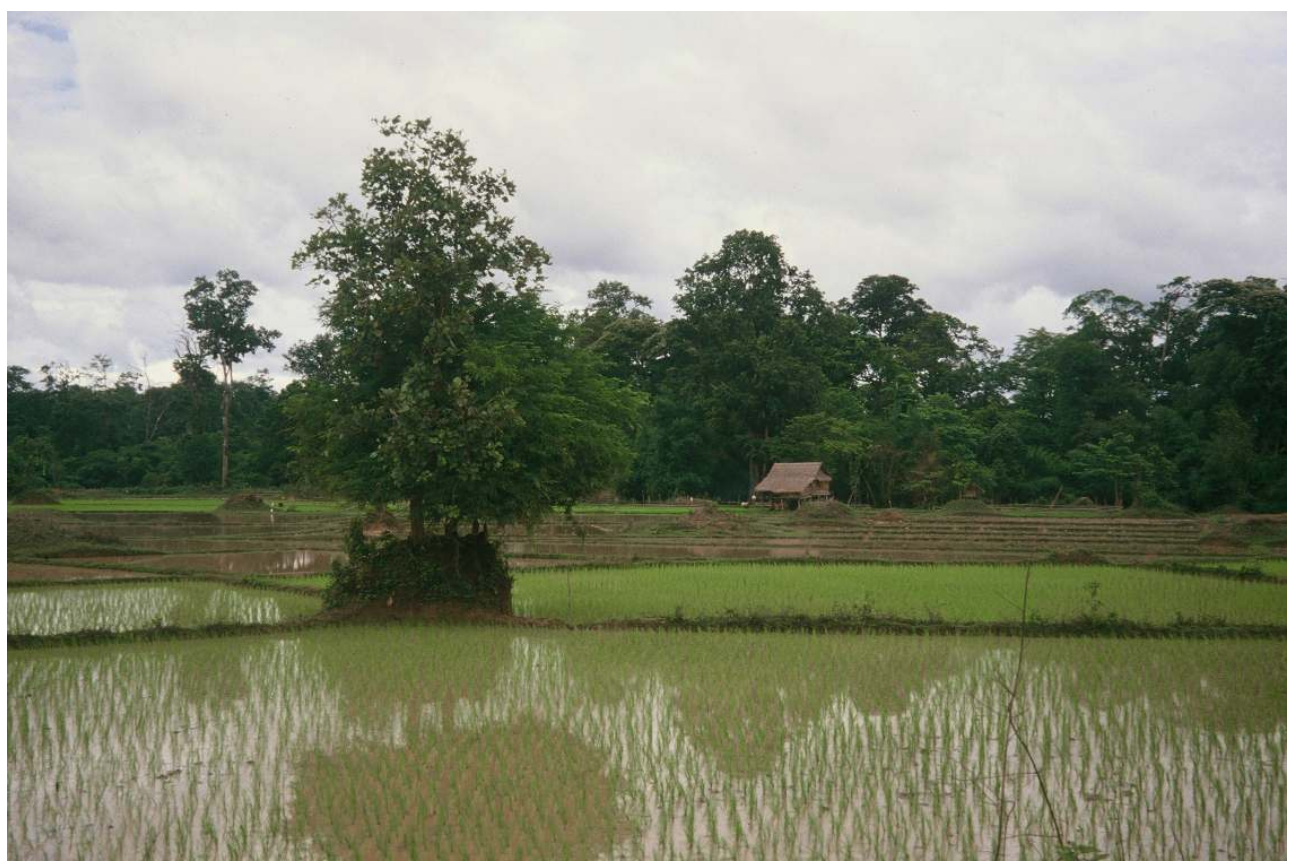

(c) F. Strigler

\section{Les menaces qui pèsent sur les ressources}

Un certain nombre d'évolutions font actuellement peser des menaces sur les ressources alimentaires disponibles dans les milieux naturels.

De nombreuses espèces animales sont menacées d'extinction depuis un demi-siècle, en raison de l'utilisation d'armes à feu et d'explosifs pendant et après les guerres d'Indochine, mais aussi et surtout, actuellement, du commerce d'animaux sauvages pour l'alimentation et la médecine traditionnelle. D'une part, l'intégration croissante de la population laotienne dans le système monétaire incite les villageois à vendre les produits de la chasse qui étaient autrefois destinés principalement à l'autoconsommation à l'exception des dons rituels ou des paiements de tribut (Mittelman et al 1997, Duckworth et al 1999). D'autre part, des chasseurs viennent des pays voisins pour capturer du gibier qu'ils revendent illégalement dans leur pays ou à des pays tiers (Srikosamatara et al 1992, Singh 2008).

Des lois limitant la chasse et le commerce des animaux sauvages ont été édictées au Laos à partir de 1986. La détention d'armes à feu est contrôlée. Dans certains districts, un permis est requis. Dans d'autres, les armes ont été complètement confisquées et seul le milicien de chaque village est autorisé à détenir un fusil. La chasse au gros gibier s'en est trouvée limitée.

En 1993 ont été mises en place des zones naturelles protégées ou NBCA (National Biodiversity Conservation Areas), qui sont au nombre de 20 et représentent au total $14 \%$ de la surface du pays (Robichaud et al 2001, Bugna 2002). D'autre part, la Convention on International Trade in Endangered Species (CITES), l'un des traités internationaux les plus importants dans le domaine de la préservation des espèces, a été signée par le Laos en 
2004. Mais, comme souvent dans les pays tropicaux, les politiques de protection de l'environnement sont basées sur une conception occidentale des relations entre l'homme et la nature, qui est fondamentalement différente des conceptions locales, ce qui ne facilite pas l'appropriation de ces politiques par les populations concernées (Instone 2003). Alors que les règles de la CITES distinguent plusieurs catégories d'espèces en fonction de leur degré de risque d'extinction, les discours ont tendance à diaboliser la chasse d'animaux sauvages en général, ce qui conduit à dénigrer et marginaliser les populations rurales dont la chasse fait partie des moyens de subsistance. Un autre problème est la difficulté d'application des réglementations, que ce soit à l'intérieur du pays ou au niveau transfrontalier. Bien que les pays voisins aient tous signé la CITES, leurs importations d'espèces interdites en provenance du Laos, dorénavant illégales, continuent comme par le passé (Singh 2008, Nguyen 2008). De plus, la construction de routes traversant le Laos d'est en ouest et du nord au sud facilite l'accès des zones forestières aux chasseurs et les débouchés aux trafiquants (Butler 2009).

Les organisations écologistes encouragent le respect des réglementations en diffusant des posters représentant les espèces en danger d'extinction et, dans les villes, des affiches alertant les consommateurs et notamment les touristes, de ne pas commander de plats de gibier dans les restaurants.

Un processus nouveau ${ }^{7}$, beaucoup plus efficace semble-t-il, est mis en œuvre dans quelques régions, notamment Bokeo et Attapeu. Les bonzes, en collaboration avec les autorités locales, organisent une cérémonie de sacralisation d'un espace naturel, forêt ou plan d'eau. On parle de bouat pa (bouat = consécration ; $p a=$ forêt). Il m'a été rapporté le $^{2}$ cas d'un villageois qui avait enfreint la règle en chassant un sanglier et qui fut blessé dans la forêt, à l'endroit même où s'était déroulée la cérémonie. Cet incident a renforcé la croyance de la population locale dans la nécessité de respecter le lieu et sa faune.

Le Laos étant l'un des derniers pays asiatiques à posséder encore des arbres de haute valeur, il attise les convoitises. L'exploitation du bois, en partie illégale, est l'une des principales causes de la déforestation. La construction de routes, liée à la mise en place de barrages ou de mines, facilite l'accès à de nouvelles zones forestières et en conséquence l'abattage illégal du bois (Lang 2001, MAF/STEA 2003). Depuis 1996, des mesures ont été prises par le gouvernement pour le limiter, mais leur application est difficile (Chape 1996, EIA/Telepak 2008).

26 L'irrigation des rizières se développe au Laos, afin de permettre une deuxième récolte de riz ou la culture de légumes. Par ailleurs, plusieurs dizaines de barrages hydroélectriques sont en cours de construction ou déjà en service, sachant que l'exportation d'électricité représente pour le Laos une des principales sources de devises. Pour l'irrigation comme pour les barrages, il est possible de minimiser l'impact sur les populations de poissons lorsqu'on le prend en compte dans les études préalables et dans la conception des installations, mais ce n'est pas toujours le cas (Chape 1996, Ratner et al 2004).

27 Au Laos, l'utilisation de pesticides reste limitée, notamment parce que les revenus des paysans ne leur permettent pas de les acheter. Elle commence néanmoins à augmenter. On a pu constater au Vietnam, en Malaisie et en Thailande, que l'utilisation de ces produits conduit à une diminution des populations d'espèces aquatiques dans les écosystèmes rizicoles (Nurhasan 2008). La destruction de la biodiversité par certaines pratiques agricoles comme l'usage de pesticides crée un cercle vicieux qui porte atteinte en retour à l'agriculture, car les espèces sauvages y jouent leur rôle : les insectes et autres 
animaux contribuent à la reproduction des espèces cultivées, à la fertilité des sols, à la régulation des espèces nuisibles.

On voit se développer au Laos de grandes plantations arboricoles, au détriment des écosystèmes traditionnels et de la biodiversité. Les premières ont été créées dans les années 1990 avec l'eucalyptus pour la production de pâte à papier. Actuellement, ce sont des milliers d'hectares qui sont concédés par le gouvernement pour la production de caoutchouc, d'huile de palme ou d'agrocarburants dans le Sud, de thé ou de canne à sucre dans le Nord. La façon dont les paysans sont dédommagés pour leurs terres montre que l'importance des ressources forestières n'est pas prise en compte : si les tecks coupés sont indemnisés à l'unité, quoique à un prix très inférieur à celui du marché, en revanche les produits de la forêt (utilisés pour l'autoconsommation, le marché local, voire l'exportation vers la Thaïlande) ne font l'objet d'aucune indemnisation car il ne s'agit pas de plantes cultivées (Luangaramsi et al 2008).

Ces plantations étaient présentées au départ comme une alternative à la déforestation liée à l'essartage et comme une source de création d'emplois en milieu rural (Alton et al 2005, Shi 2008). Actuellement, le gouvernement commence à prendre conscience de leurs effets délétères et y met un frein (Vientiane Times 2008), même si ces décisions ne sont pas toujours suivies d'effet au niveau provincial (Hanssen 2007).

\section{L'accès aux ressources}

Les villages situés dans des zones forestières éloignées des routes, sont souvent considérés comme défavorisés et pauvres, n'ayant qu'un accès très limité aux structures de soins, d'éducation et de commerce. Des études montrent cependant une meilleure situation nutritionnelle dans ces villages, liée à un meilleur accès aux produits animaux et végétaux sauvages. Par exemple Krahn (2005), dans sa thèse sur l'alimentation des villages katu de la province de Sékong observe que les villages en zones forestières bénéficient de meilleurs apports nutritionnels que les villages plus proches des zones urbaines. Murayama et al (2007) font la même constatation dans la province de Savannakhet.

31 Face à la question de la sécurité alimentaire, les solutions proposées sont souvent inadaptées, comme l'intégration au marché ou l'augmentation de la production agricole, qui s'avèrent inefficaces (Krahn 2005), voire contre-productives (Ducourtieux 2006). Les paysans, encouragés à produire plus de riz et à élever des animaux, n'ont plus le temps de chasser et de récolter les produits de la forêt. Or, les produits de la forêt, par leur diversité, jouent un rôle important dans la couverture des besoins en micronutriments, et ils constituent des réserves de nourriture beaucoup plus fiables que les cultures, qui sont à la merci des accidents climatiques, et que les élevages, susceptibles d'être atteints de maladies (Krahn 2005).

Depuis plus d'un siècle, l'agriculture d'abattis-brûlis, ou essartage, est activement combattue, dans les pays où elle existe encore, et fait l'objet de débat parmi les scientifiques. Le gouvernement laotien, encouragé en cela par certaines ONG et organisations internationales, a décrété l'éradication de ce système d'agriculture. Or, sa complexité et sa logique sont largement méconnues hormis des populations qui le pratiquent, et il permet dans certains cas de préserver la biodiversité et d'assurer la satisfaction des besoins des populations locales (Svengsuksa 2003, McNeely 2004, 
Ducourtieux 2006). Le problème est que cette agriculture évolue vers des pratiques moins durables, c'est-à-dire une rotation plus rapide des parcelles avec en conséquence un raccourcissement des durées de friche, ou une culture des parcelles pendant plus de deux années consécutives, d'où un épuisement des sols, un déclin de la productivité et une invasion par des adventices invasives. Cette évolution peut être due à la croissance démographique, mais elle est paradoxalement en grande partie initiée par les réglementations qui, en vue d'éradiquer ce type d'agriculture, limitent les surfaces autorisées (Vandergeest 2003, ADB 2008).

Une réforme foncière a débuté dans les années 1990, afin d'améliorer l'accès à la terre pour les paysans pauvres et d'assurer la sécurité foncière en clarifiant les droits de propriété. Elle vise aussi à consolider le contrôle de l'État sur les terres et à pousser les paysans à renoncer à l'essartage (Vandergeest 2003, Evrard 2004, ADB 2008). De plus en plus de villages situés dans les zones de montagnes et de forêts sont amenés à se déplacer, soit volontairement, soit sous la pression des autorités, pour être relocalisés à proximité de routes. Certes, ces déplacements facilitent l'accès de la population aux structures de soins, d'éducation et de commerce. Mais ils conduisent parfois à une aggravation des problèmes des paysans, notamment sur le plan nutritionnel, les systèmes alimentaires se trouvant modifiés. Ces modifications peuvent avoir des aspects positifs ou négatifs selon les cas. Malheureusement, les aspects négatifs sont insuffisamment pris en compte par les autorités et les institutions agissant dans le domaine de la sécurité alimentaire. De mauvais postulats (les villages les plus reculés sont les plus touchés par la pauvreté, la maladie et la malnutrition) conduisent à des décisions inadaptées (déplacer les villages) qui renforcent des problèmes comme l'isolement (de plus en plus de villages étant déplacés, ceux qui restent en altitude se retrouvent de plus en plus isolés), les problèmes de santé (populations arrivant dans des zones impaludées alors qu'elles vivaient dans des zones montagnardes épargnées par ce fléau), les problèmes alimentaires (les populations déplacées se retrouvent dans un environnement qu'elles ne connaissent pas et dont elles ont plus de difficulté à exploiter les ressources) (Goudineau 1997).

Les politiques agricoles et foncières encouragent les populations montagnardes à intensifier leur production et à développer des cultures commerciales, mais le manque d'infrastructures et d'accès au marché dans les zones reculées ne permet pas ces développements et les populations de ces zones se trouvent de plus en plus marginalisées à cause de la restriction de l'essartage qu'on leur impose (Ducourtieux et al 2004). La réduction des surfaces d'abattis-brûlis et corrélativement des temps de friche, entraîne une diminution de la fertilité des champs et de la production de paddy. Le recours aux produits forestiers s'en trouve accru, ce qui, combiné avec la limitation des espaces forestiers accessibles, conduit à une raréfaction des ressources (Evrard 2004, ADB 2008). Cette situation semble justifier le bien-fondé des politiques mises en œuvre, dans un cercle vicieux conduisant à renforcer les problèmes nutritionnels (Krahn 2005).

Le gouvernement laotien aspire à faire sortir le Laos du groupe des PMA, et s'est fixé pour cela les objectifs suivants : accélération de la croissance, dynamisation du commerce international et de l'investissement, promotion du secteur privé, réduction de la pauvreté dans les zones rurales prioritaires. Les ONG et organisations internationales mettent en place des projets destinés à "réduire la pauvreté", avec entre autres moyens, la mise en place d'activités génératrices de revenus et la facilitation de l'accès au marché. Sans entrer dans le débat du bien-fondé de ces projets, on peut constater que l'un des effets pervers est de détourner vers le marché une partie des produits alimentaires qui étaient 
autrefois consommés par les villageois (Bouahom et al 2004, Singh 2008). La commercialisation de produits forestiers est à double tranchant pour les populations locales: elle constitue une source de revenus, mais peut porter atteinte à leur alimentation si elle entre en compétition avec celle-ci (Bush 2004, Krahn 2005, Colfer et al 2006).

Les politiques de développement qui encouragent les villageois à cultiver leur apportent certes des revenus monétaires qu'ils utilisent pour acheter la nourriture qu'auparavant ils collectaient gratuitement dans la forêt, mais elles n'augmentent pas forcément leur niveau de vie ni leur état nutritionnel. Delang (2006) a montré, pour les Karen de Thaillande, que l'achat d'aliments sur le marché, pour fournir une alimentation nutritionnellement équivalente à celle qu'ils ont actuellement grâce à la cueillette, leur demanderait environ dix fois plus de temps de travail. De plus, il faut considérer le fait que l'agriculture correspond à un travail alors que la chasse et la cueillette sont plutôt apparentées à un loisir (Schlemmer 1999).

\section{Conclusion}

37 La place de la chasse, la pêche et la cueillette dans l'alimentation des Laotiens est encore très importante, mais elle est en train de diminuer pour diverses raisons liées à l'évolution de la société (nouvelles techniques agricoles, adaptation au système monétaire, etc.) et aux politiques mises en œuvre (éradication de l'abattis-brûlis, déplacements de population, réglementation de la chasse). Ces évolutions bouleversent les systèmes alimentaires: l'accès aux ressources naturelles se trouve limité, sans que l'accès au marché soit possible pour la majorité. La situation nutritionnelle s'en trouve dégradée, sauf dans les villes, où l'offre alimentaire est plus variée et accessible, mais où commencent à apparaître les effets délétères de la transition nutritionnelle.

L'ensemble des problématiques évoquées montre que la question de la couverture des besoins alimentaires et celle de la biodiversité sont dépendantes de facteurs environnementaux, culturels, sociaux, économiques et politiques. Les professionnels de l'écologie et ceux du développement ont souvent poursuivi des objectifs conflictuels, et le coût en terme nutritionnel de la dégradation de l'environnement a rarement été pris en compte. Des projets plus intégrés sont actuellement mis en œuvre (Aubertin 2000, Foppes \& Dechaineux 2000, Chape 2001, Butler 2009), qui cherchent à concilier impératifs économiques et écologiques : systèmes locaux de gestion communautaire des ressources aquatiques ou forestières, systèmes de domestication de certaines espèces végétales dans des "jardins" forestiers, écotourisme. Le respect du mode de vie des populations locales est primordial, et on a vu combien les représentations de concepts comme la nature, la biodiversité, le développement, sont des constructions sociales qui sous-tendent des enjeux politiques et économiques allant de l'échelle locale à l'échelle internationale.

Par le passé, la biodiversité a constitué une "garantie" permettant de faire face aux besoins alimentaires même en cas d'accidents climatiques affectant les cultures. Aujourd'hui, avec l'intégration au marché, elle joue aussi un rôle de "garantie" par rapport à un autre type d'accidents, liés aux fluctuations du marché. 


\section{BIBLIOGRAPHIE}

ADB 2008 - Lao PDR: Institutional Strengthening for Poverty Monitoring and Evaluation. Manille, Asian Development Bank, 103 p.

Alton C., Bluhm D. \& Sannanikone S. 2005 - Rubber survey in Luang Namtha Province, 112 p.

Aubertin C. 2000 - Les systèmes intermédiaires : un concept pour le développement durable?

Quelques exemples au Brésil et au Laos. In Cultivating (in) tropical forests: The evolution and sustainability of intermediate systems between extactivism and plantations. Lofoten, Norway, FOREASIA.

Barrau J. 1974 - L'Asie du Sud-Est, berceau cultural. Études rurales, 53-56 : 17-39.

Bouahom B., Douangsavanh L. \& Rigg J. 2004 - Building sustainable livelihoods in Laos: untangling farm from non-farm, progress from distress. Geoforum, 35 : 607-619.

Boulbet J. 1984 - Forêts et pays, EFEO, 134 p.

Bugna S. 2002 - A Profile of the Protected Area System of Lao PDR, 46-52.

Burlingame B. 2000 - Wild nutrition. J Food Comp Anal, 13 : 99-100.

Bush S. 2004 - Scales and sales: Changing social and spatial fish trading networks in the Siiphandone fishery, Lao PDR, Singapore Journal of Tropical Geography, 25(1) : 32-50.

Butler R. 2009 - Laos Emerges as Key Source. In Asia's Illicit Wildlife Trade, Yale Environment 360.

Chape S. 1996 - Biodiversity conservation, protected areas and the development imperative in lao PDR; forging the links, IUCN.

Chape S. 2001 - An overview of integrated approaches to conservation and community development in the Lao People's Democratic Republic. Parks, 11(2) : 24-32.

Chazée L. 1998 - Évolution des systèmes de production ruraux en République Démocratique Populaire du Laos (1975-1995). L'Harmattan, 429 p.

Chazée L. 1990 - The Mammals of Laos and the Hunting Practices. Vientiane, Private report.

Clendon K. 2001 - The Role of Forest Food Resources in Village Livelihood Systems: A Study in Three Villages in Salavan Province, Lao PDR. Vientiane, Non-Timber Forest Products Project in Lao PDR, MAF/IUCN/WCU, $41 \mathrm{p}$.

Colfer CJP, Sheil D., Kaimowitz D. \& Kishi M. 2006 - Les forêts et la santé humaine dans les tropiques : quelques interactions importantes. Unasylva, 57(2) : 3-10.

Davidson A. 1975 - Fish and fish dishes of Laos, Tokyo and Rutland, Vermont, Ed. Charles Tuttle.

DeFoliart G.R. 1997 - An overview of the role of edible insects in preserving biodiversity. Ecol. Food. Nutr., $36: 109-132$.

Delang C. 2006 - The role of wild food plants in poverty alleviation and biodiversity conservation in tropical countries. Progress in Development Studies, 6 : 275-286.

Doré A. 1996 - L'école de la forêt. Un itinéraire spirituel lao, Kailash, 138 p. 
Dounias E. \& Froment A. 2006 - Lorsque les chasseurs-cueilleurs deviennent sédentaires : les conséquences pour le régime alimentaire et la santé. Unasylva, 57(2) :26-33.

Duckworth W., Salter R. \& Khounboline K. 1999 - Wildlife in the Lao PDR. 1999 Status Report, Vientiane, IUCN, CPAWM, WCS.

Ducourtieux O., Laffort J.-R \& Sacklokham S. 2004 - La réforme foncière au Laos. Une politique hasardeuse pour les paysans, Revue Tiers Monde, XLV, 177 : 207-229.

Ducourtieux 0. 2006 - Du riz et des arbres : L'élimination de l'agriculture d'abattis-brûlis, une constante politique au Laos, Thèse de doctorat, Paris, INA P-G, 635 p.

EIA/Telepak 2008 - Borderlines. Vietnam's Booming Furniture Industry and Timber Smuggling in the Mekong Region, $20 \mathrm{p}$.

Emerton L. 2003 - Forest environmental values and socio-economic development: a case study of Lao PDR, XIIe congrès forestier mondial, Québec.

Etkin N. \& Ross P. 1994 - Pharmacologic Implications of 'Wild' Plants in Hausa Diet. In Etkin N.L. (Ed.), Eating on the Wild Side. The Pharmacologic, Ecologic, and Social Implications of Using Noncultigens. Tucson, University of Arizona Press : 85-101.

Evrard 0. 2004 - La mise-en-œuvre de la réforme foncière au Laos, FAO, 48 p.

FAO, 2005 - La situation des forêts dans le monde.

Foppes J. \& Dechaineux R. 2000 - Non-Timber Forest Products in Lao PDR: a practical application of the ecosystems approach? Case-study for the pathfinder workshop "An Ecosystem Approach under the Convention on Biological Diversity; from concept to action", Malaysia, $15 \mathrm{p}$.

Foppes J. \& Ketphan S. 2000 - Forest extraction or cultivation? Local solutions from Lao PDR, FOREASIA Workshop.

Frison E., Smith I.F., Johns T., Cherfas J. \& Eyzaguirre P. 2005 - Using biodiversity for food, dietary diversity, better nutrition and health. SAJCN, 18(2) :112-114.

Goudineau Y. 1997 - Resettlement and social characteristics of new villages. Vientiane, UNDP, vol. 1.

Grivetti L. \& Ogle B. 2000 - Value of traditional foods in meeting macro- and micronutrient needs: the wild plant connection. Nutrition Research Reviews, $13: 36-41$.

Halwart M. 2006 - Biodiversity and nutrition in rice-based aquatic rice-based ecosystems. J. Food Composition and Analysis, 19(6-7) : 747-751.

Hanssen C. [2007] - Lao land concessions, development for the people? Presented at: International Conference on Poverty Reduction and Forests : Tenure, Market and Policy Reforms, RECOFTC, Bangkok, 3-7 Sept 2007.

Hladik A., Leigh E.T \& Bourlière F. 1993 - Food production and nutritional value of wild and semidomesticated species - Background. In Hladik C.M., Hladik A., Linares O., Pagézy H., Semple A., Hadley M. (Ed.), Tropical forests, people and food. Paris, UNESCO/The Parthenon Publishing Group : 127-138.

Instone L. 2003 - T(r)opical Translations: Reterritorialising the Space of Biodiversity Conservation, RMAP Working Paper $n^{\circ} 46,18 \mathrm{p}$.

Johns T. 2003 - Plant biodiversity and malnutrition: simple solutions to complex problems. Afr. J. Food Agric. Nutr. Dev., $3:$ 45-52.

Johns T. \& Sthapit R. 2004 - Biocultural diversity in the sustainability of developing-country food systems. Food Nutr. Bull., 25(2) : 143-155. 
Kottelat M. 2001 - Fishes of Laos. Colombo, Wildlife Heritage Trust, 198 p.

Krahn J. 2005 - The dynamics of dietary change of transitional food systems in tropical forest areas of Southeast Asia. The contemporary and traditional food system of the Katu in the Sekong Province, Lao PDR, Thèse de doctorat, Rheinischen Friedrich-Wilhelms-Universität, Bonn, 172 p.

Kuhnlein H. \& Receveur O. 1996 - Dietary change and traditional food systems of indigenous peoples. Ann. Rev. Nutr., $16: 417-442$.

Kuhnlein H. 2003 - Micronutrient nutrition and traditional food systems of indigenous peoples, Food. Nutrition and Agriculture, FAO, 32 : 33-39.

Lang C. 2001 - Deforestation in Vietnam, Laos and Cambodia. In Vajpeyi DK (Ed.), Deforestation, Environment, and Sustainable Development : A Comparative Analysis, Praeger, Westport and London : 111-137.

Luangaramsi P., Leonard R. \& Kuaycharoen P. 2008 - Socio-economic and Ecological implications of large scale industrial plantations in the Lao PDR, Case Study on Rubber Plantation, Chiang Mai University.

MAF/STEA 2003 - Biodiversity Country Report, Vientiane, 169 p.

McNeely J. 2004 - Nature vs. nurture : managing relationship between forests, agroforestry and wild biodiversity. Agroforestry Systems, 61: 155-165.

Michon G. 2004 - Cultiver la forêt dans les systèmes paysans en Asie du Sud-est. Comptes-rendus de l'Académie d'Agriculture de France, 90(3) : 87-88.

Mittelman A.J., Lai Chun K., Byron N., Michon G. \& Katz E. 1997 - Non-wood forest products outlook study for Asia and the Pacific: Towards 2010, FAO, Working Paper n APFSOS/WP/28, 69 p.

Murayama N. \& Natsuhara K. [2007] - Nutrition Ecological Study to improve Maternal and Child Health in Lao PDR, National Health Research Forum to Promote the Health Research Systems Strengthening in Lao PDR, Vientiane, $24^{\text {th }}-25^{\text {th }}-26^{\text {th }}$ September 2007.

Nguyen V.S. 2008 - Wildlife Trading in Vietnam - Situation, Causes, and Solutions. The Journal of Environment \& Development, 17(2) : 145-164.

Nurhasan M. 2008 - Nutritional composition of aquatic species in Laotian rice field ecosystems: possible impact of reduced biodiversity, Thèse, Université de Tromso.

Ogle B., Hung P.H. \& Tuyet H.T. 2001 - Significance of wild vegetables in micronutrient intakes of women in Vietnam: an analysis of food variety. Asia Pacific J Clin Nutr, 10(1) : 21-30.

Pagézy H. 1993 - The importance of natural resources in the diet of the young child in a flooded tropical forest in Zaire. In Hladik C.M., Hladik A., Linares O., Pagézy H., Semple A. \& Hadley M. (Ed.), Tropical forests, people and food, Paris, UNESCO/The Parthenon Publishing Group : 365-380.

Phongphit S. 1995 - Savoirs populaires et développement rural, FPH, Dossier pour un débat nº 43, 140 p.

Ratner B., Ha D.T., Kosal M., Nissapa A. \& Chaphengxay S. 2004 - Undervalued and Overlooked: Sustaining Rural Livelihoods through Better Governance of Wetlands, WorldFish Center Studies and Reviews, 28.

Robichaud W., Marsh CW., Southammakoth S. \& Khounthikoummane S. 2001 - Review of the national protected area system of Lao PDR, Vientiane, Lao-Swedish Forestry Programme, 133 p. Schlemmer G. 1999 - Utilisation des produits de chasse et de cueillette dans le district de Viengthong, province de Houaphan, Étude préliminaire. Vientiane, IRD, Faculté d'agriculture et des forêts. 
Shi W. 2008 - Rubber Boom in Luang Namtha. A Transnational Perspective, GTZ, 75 p.

Singh S. 2008 - Contesting moralities: the politics of wildlife trade in Laos. Journal of Political Ecology, 15 : 1-20.

Sisouphanthong B. \& Taillard C. 2000 - Atlas de la République démocratique populaire lao, Paris, CNRS-Libergéo/La Documentation française, $160 \mathrm{p}$.

Srikosamatara S., Siripholdej B. \& Suteethorn V. 1992 - Wildlife trade in Lao PDR and between lao PDR and Thailand. Natural History Bulletin of the Siam Society, 40(1) : 1-47.

Svengsuksa B. 2003 - Reconstitution du couvert végétal et revalorisation des terrains après la culture sur brûlis dans le district de Muang Fuang, province de Vientiane, RDP Lao, Thèse pour obtenir le grade de docteur au Muséum national d'histoire naturelle, $128 \mathrm{p}$.

Taillard C. 1977 - L'espace social : quelques réflexions à propos de deux exemples au Laos, ASEMI, VIII : 81-102.

Vandergeest P. 2003 - Affectation des terres et déplacements de population induits par le développement au Laos, RISS, 175 (1) : 49-59.

Vidal J. 1963 - Les plantes utiles du Laos, JATBA.

Vientiane Times, Ministry protects forests from rubber plantations, by Somsack Pongkhao, 18 Nov 2008.

\section{NOTES}

1. Cette diversité est mal connue d'un point de vue scientifique. Pour les végétaux, un important travail de répertorisation a été effectué par Jules Vidal (1963) dans les années 1950. Pour les animaux, les travaux sont plus récents (Chazée 1990, Duckworth et al. 1999) et de nouvelles espèces sont régulièrement identifiées.

2. La population laotienne est constituée d'une cinquantaine de groupes ethniques, qui se partagent en quatre familles ethnolinguistiques : taï-kadaï (Lao, Lu, Taï dam...), austro-asiatique (Khamu, Katu...), miao-yao (Hmong, Yao...) et sino-tibétaine (Phounoy, Ho...). Les Taï-Kadaï vivent principalement dans les fonds de vallées et cultivent essentiellement des rizières, alors que les trois autres catégories de population vivent plutôt dans les zones d'altitude et pratiquent, pour certaines, l'essartage (Sisouphanthong \& Taillard 2000).

3. Ceci est caractéristique des "écosystèmes généralisés", dont font partie la plupart des écosystèmes forestiers tropicaux (Barrau 1974).

4. Ce chiffre donne une idée de la situation en comparaison avec les autres pays, mais est à considérer avec prudence car il varie selon les sources, en fonction notamment des définitions de la forêt.

5. Cette conception n'est peut-être pas celle de tous les groupes ethniques, mais elle existe notamment chez les Lao.

6. Pas toujours distincts du précédent.

7. Il s'agit d'un processus nouveau au Laos, mais qui existait en Thaïlande. Voir par exemple Phongphit (1995).

8. Entretien avec Mme N le 6 janvier 2009. 


\section{RÉSUMÉS}

Le Laos compte 6 millions d'habitants, dont 80 \% vivent en milieu rural. En accompagnement du riz, qui forme la base de leur alimentation, les Laotiens consomment une très grande variété de produits végétaux et animaux, dont une part importante provient de la cueillette, la pêche et la chasse. Le recours à ces ressources naturelles permet de varier les apports alimentaires, mais aussi de les rendre suffisants pendant les périodes de l'année où les cultures ne le permettent pas. Dans le contexte actuel de transition économique et de nouvelles pratiques agricoles, la disponibilité et l'accessibilité de ces ressources se trouvent affectées.

There are 6 million inhabitants in Laos, $80 \%$ of whom live in rural areas. Together with rice, which is their staple diet, Laotians consume a great variety of vegetable and animal products, obtained through gathering, fishing, and hunting. Those natural resources enable them to vary their food intake, but also to complement it during the periods of the year when their crops are insufficient. Within the current context of economic transition and new farming practices, the availability and accessibility of those resources are affected.

\section{INDEX}

Mots-clés : sécurité alimentaire, produits forestiers non ligneux

Keywords : food security, Lao, non timber forest products

Index géographique : Laos

\section{AUTEUR}

\section{FLORENCE STRIGLER}

Fonds français pour l'alimentation et la santé

42 rue Scheffer

75116 Paris

f.strigler@alimentation-sante.org 